TITLE:

\title{
Nonadiabatic coupling between C- $O$ stretching and Pt substrate electrons enhanced by frustrated mode excitations
}

\section{$\operatorname{AUTHOR}(\mathrm{S}):$}

Watanabe, Kazuya; Inoue, Ken-ichi; Nakai, Ikuyo F.; Matsumoto, Yoshiyasu

\section{CITATION:}

Watanabe, Kazuya ... [et al]. Nonadiabatic coupling between C-O stretching and Pt substrate electrons enhanced by frustrated mode excitations. Physical Review B 2010, 81(24): 241408.

\section{ISSUE DATE:}

2010-06

URL:

http://hdl.handle.net/2433/128893

RIGHT:

(C) 2010 The American Physical Society 


\title{
Nonadiabatic coupling between $\mathrm{C}-\mathrm{O}$ stretching and Pt substrate electrons enhanced by frustrated mode excitations
}

\author{
Kazuya Watanabe, ${ }^{1,2, *}$ Ken-ichi Inoue, ${ }^{1}$ Ikuyo F. Nakai, ${ }^{1}$ and Yoshiyasu Matsumoto ${ }^{1,3}$ \\ ${ }^{1}$ Department of Chemistry, Graduate School of Science, Kyoto University, Kyoto 606-8502, Japan \\ ${ }^{2}$ PRESTO, JST, 4-1-8 Honcho Kawaguchi, Saitama, Japan \\ ${ }^{3}$ Institute for Molecular Science, Okazaki, Aichi 444-8585, Japan \\ (Received 5 April 2010; revised manuscript received 20 May 2010; published 9 June 2010)
}

\begin{abstract}
We report the transient enhancement of coupling between adsorbate internal stretching vibration and substrate electrons induced by femtosecond laser excitation. A Pt(111) surface covered with carbon monoxide (CO) is irradiated with a $150 \mathrm{fs}$ pump pulse at $400 \mathrm{~nm}$. Transient changes in the imaginary part of nonlinear susceptibility in the $\mathrm{C}-\mathrm{O}$ stretching region are probed by using femtosecond time-resolved infrared-visible sum-frequency generation with phase-sensitive detection. The line shape of C-O stretching shows remarkable asymmetry due to Fano interference with a continuum of electron-hole pair excitation. Excitations of CO-Pt frustrated modes play a key role in the enhancement of the nonadiabatic coupling.
\end{abstract}

DOI: 10.1103/PhysRevB.81.241408

Vibrational energy transfer is an important factor controlling elementary processes at surfaces, including adsorption, diffusion, reaction, and desorption of adsorbate. At metal surfaces, nonadiabatic coupling between adsorbate vibration and substrate electrons becomes indispensable particularly for a vibrational mode with a frequency higher than a substrate phonon continuum band. ${ }^{1,2}$ A typical example is the internal stretching (IS) of carbon monoxide (CO) adsorbed on metals. Its vibrational lifetime is drastically shortened to a few picosecond when $\mathrm{CO}$ is adsorbed on metal surfaces; 3,4 the effective damping was accounted for by a nonadiabatic decay via substrate electron-hole pair (EHP) excitations., ${ }^{2,5,6}$

Excitations with ultrashort laser pulses have opened a way to investigate the nonadiabatic effects under strong nonequilibrium conditions between substrate electrons and adsorbates. ${ }^{7}$ Under such conditions, an IS band transiently redshifts and broadens, ${ }^{8-13}$ these features are attributed to pure dephasing caused by anharmonic couplings of the IS mode to frustrated translation (FT) and frustrated rotation (FR) modes. ${ }^{14}$ Moreover, adsorbates effectively diffuse and desorb, and the yields of these processes increase nonlinearly with pump pulse fluence. ${ }^{15-20}$ From these observations, a picture has emerged on the coupling of adsorbate nuclear motions with substrate electrons: substrate hot electrons excite effectively center-of-mass (COM) motions of adsorbates, leading diffusion, desorption, and reaction. ${ }^{21}$ Despite this widely accepted picture, it is largely unknown how the nonadiabatic coupling strength of the IS mode to substrate electrons varies under the strong nonequilibrium conditions.

Time-resolved infrared-visible sum-frequency generation (TR-IVSFG) has been used extensively for probing adsorbate vibration dynamics. ${ }^{9-13}$ The spectral line shape of an IS mode in SFG spectra contains not only the information of anharmonic couplings between the IS and adsorbatesubstrate modes but also that of nonadiabatic coupling of IS mode with substrate electrons. However, it is difficult to separate the contribution of nonadiabatic coupling from that of anharmonic couplings because the conventional homodyne detection only provides the squared modulus of a second-order susceptibility $\left|\chi^{(2)}\right|^{2}$.

To overcome this obstacle, we employed heterodyne de-
PACS number(s): 78.47.J-, 68.43.Pq, 82.53.St

tection that allows us to determine the real and imaginary parts of $\chi^{(2)}$ separately. ${ }^{22}$ We show that spectra of the imaginary part of $\chi^{(2)}$, i.e., $\operatorname{Im}\left[\chi^{(2)}\right]$, of on-top $\mathrm{CO}$ on $\mathrm{Pt}(111)$ exhibit large degrees of transient asymmetry under intense irradiation with pump pulses at $400 \mathrm{~nm}$. The asymmetry is due to the Fano interference ${ }^{23}$ between discrete vibrational levels and a substrate EHP continuum as was proposed by Langreth. ${ }^{24,25}$ The analysis of time-resolved $\operatorname{Im}\left[\chi^{(2)}\right]$ spectra enables us to clarify an effective role of COM motions of $\mathrm{CO}$ in the nonadiabatic coupling between the IS mode and platinum substrate electrons.

Figure 1(a) shows a schematic of a setup for the heterodyne detection of TR-IVSFG which was modified from a homodyne-detection version described previously. ${ }^{26}$ Experiments were performed with an ultrahigh vacuum (UHV) chamber (base pressure $<2 \times 10^{-10}$ Torr). A Pt(111) single crystal was cleaned by cycles of $\mathrm{Ar}^{+}$sputtering, annealing $(1000 \mathrm{~K})$, and oxygen treatment $\left(1 \times 10^{-7}\right.$ Torr, $\left.800 \mathrm{~K}\right)$. The output pulse of a Ti:sapphire regenerative amplifier [Spectra physics, $1 \mathrm{kHz}, 1.8 \mathrm{~mJ} /$ pulse] was split into three to generate a broad band infrared (IR) pulse (150-fs duration, $7 \mu \mathrm{J} /$ pulse, centered at $\sim 2100 \mathrm{~cm}^{-1}$ ), a narrow band 800 $\mathrm{nm}$ "visible" pulse (1-ps duration, $15 \mu \mathrm{J} /$ pulse), and a 400-nm pump pulse (150-fs duration). The $p$-polarized three beams were focused onto the sample surface by a spherical mirror. Phase sensitive detection of SFG signals was performed in a manner similar to that developed by other

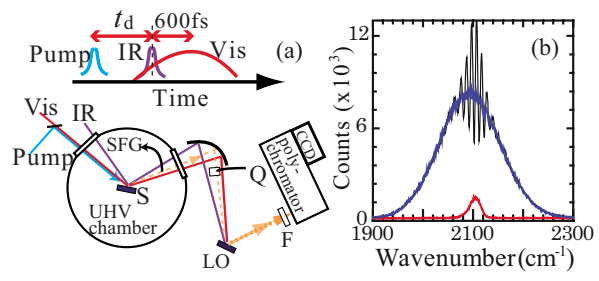

FIG. 1. (Color online) (a) A schematic of pulse timing and a setup for TR-IVSFG with heterodyne detection. LO: local oscillator, Q: quartz plate, S: sample, and F: filter. (b) SFG spectra of on-top CO on $\mathrm{Pt}(111)$ observed with heterodyne (black) and homodyne detection (red, light gray) in addition to a spectrum of LO (blue, dark gray). 
groups. ${ }^{27,28} \mathrm{SFG}$ signals from the sample surface were mixed with those from a local oscillator (LO) generated by the IR and visible pulses refocused onto a GaAs wafer placed outside the UHV chamber. A time interval between the two signals $t_{\mathrm{LO}} \sim 3 \mathrm{ps}$ was adjusted by a quartz plate inserted in the optical path of the signal beam. Spectral interferograms were recorded by using a polychromator with a chargecoupled device camera as a function of time delay of an IR pulse from a $400-\mathrm{nm}$ pump pulse $t_{\mathrm{d}}$ while a time interval between IR and visible pulses was fixed at 600 fs [see Fig. 1(a)]. The average sample temperature was kept at $90 \mathrm{~K}$ during irradiation with laser pulses while the absorbed fluence of 400-nm pump pulses $I$ was varied from 3 to $13 \mathrm{~mJ} / \mathrm{cm}^{2}$. At $I>5 \mathrm{~mJ} / \mathrm{cm}^{2}$, CO coverage was depleted by photodesorption within a few seconds. Hence, we continuously exposed a sample to $\mathrm{CO}$ at a constant pressure in the range from $3 \times 10^{-8}$ to $5 \times 10^{-7}$ Torr, depending on the fluence of pump pulses, to maintain a fractional coverage $\theta$ at $\sim 0.3$ ML during the measurements.

A typical heterodyne-detected spectrum of the IS band of on-top CO at $\theta=0.5 \mathrm{ML}$ without pump-pulse irradiation is depicted in Fig. 1(b) together with a conventional homodyne-detected one. Heterodyne-detected SFG spectra $S_{\text {tot }}(\omega)$ are given by

$$
\begin{aligned}
S_{\mathrm{tot}}(\omega) \propto & \left|E_{\mathrm{s}}(\omega)+E_{\mathrm{LO}}(\omega)\right|^{2}=\left|E_{\mathrm{s}}(\omega)\right|^{2}+\left|E_{\mathrm{LO}}(\omega)\right|^{2} \\
& +2 \operatorname{Re}\left[E_{\mathrm{s}}(\omega)^{*} E_{\mathrm{LO}}(\omega) \exp \left(i \omega t_{\mathrm{LO}}\right)\right],
\end{aligned}
$$

where $\omega$ is the sum frequency of IR $\left(\omega_{\mathrm{IR}}\right)$ and visible $\left(\omega_{\mathrm{VIS}}\right)$ photons, $E_{\mathrm{s}}(\omega)$ and $E_{\mathrm{LO}}(\omega)$ are the Fourier transformed spectra of SFG fields from the sample and the LO, respectively. From the third term in Eq. (1), one can obtain a complex electric field $A(\omega)=E_{\text {sig }}(\omega) E_{\mathrm{LO}}^{*}(\omega) \exp \left(i \omega t_{\mathrm{LO}}\right){ }^{28,29}$

Measurements with a reference sample, a GaAs wafer located at the position of the Pt crystal in the UHV chamber, provided a complex field of the reference $A_{\text {ref }}(\omega)$. Because the second-order susceptibility of the reference $\chi_{\text {ref }}^{(2)}=\left|\chi_{\text {ref }}^{(2)}\right| \exp \left(i \theta_{\text {ref }}\right)$ is almost constant in the IR frequency region measured, a ratio spectrum $r(\omega)=A(\omega) / A_{\text {ref }}(\omega)$ is proportional to $\left|\chi_{\mathrm{s}}^{(2)}(\omega)\right| \exp \left[i \theta_{\mathrm{s}}(\omega)-i \theta_{\mathrm{ref}}\right]$, where $\chi_{\mathrm{s}}^{(2)}(\omega)$ $=\left|\chi_{\mathrm{s}}^{(2)}(\omega)\right| \exp \left[i \theta_{\mathrm{s}}(\omega)\right]$ is the nonlinear susceptibility of sample. We chose the unknown parameter $\theta_{\text {ref }}$ in such a way that the phase of $r(\omega)$ becomes zero at the lower frequency side of CO stretching band $\left(2000-2050 \mathrm{~cm}^{-1}\right)$ under no pump irradiation.

Figure 2 shows a series of imaginary part of $r(\omega)$ as a function of $t_{\mathrm{d}}$ together with a real part at $t_{\mathrm{d}}=-10 \mathrm{ps}$. Here, we present spectra with respect to $\omega_{\mathrm{IR}}$ by subtracting $\omega_{\mathrm{VIS}}$ from $\omega$. Modulation in each spectrum at $-2.0<t_{\mathrm{d}}<0 \mathrm{ps}$ is attributed to a perturbed free induction decay induced by 400-nm pump pulses; C-O coherent oscillation was affected by a delayed pump pulse. At $0<t_{\mathrm{d}}<3 \mathrm{ps}$, the peak redshifted and decreased in amplitude; these are consistent with the earlier works. ${ }^{8,10,11}$ What is remarkable is that the $\operatorname{Im}[r(\omega)]$ spectra at $0<t_{\mathrm{d}}<10$ ps show appreciable asymmetric line shapes.

The nonlinear susceptibility of sample $\chi_{\mathrm{s}}^{(2)}$ is composed of a vibrationally nonresonant $\chi_{\mathrm{NR}}^{(2)}$ and a resonant term $\chi_{\mathrm{R}}^{(2)}$ $=(A \alpha \mu) /\left(\omega_{\mathrm{IR}}-\omega_{0}+i \gamma\right)$, where $A$ is a constant; $\alpha, \mu, \omega_{0}$, and

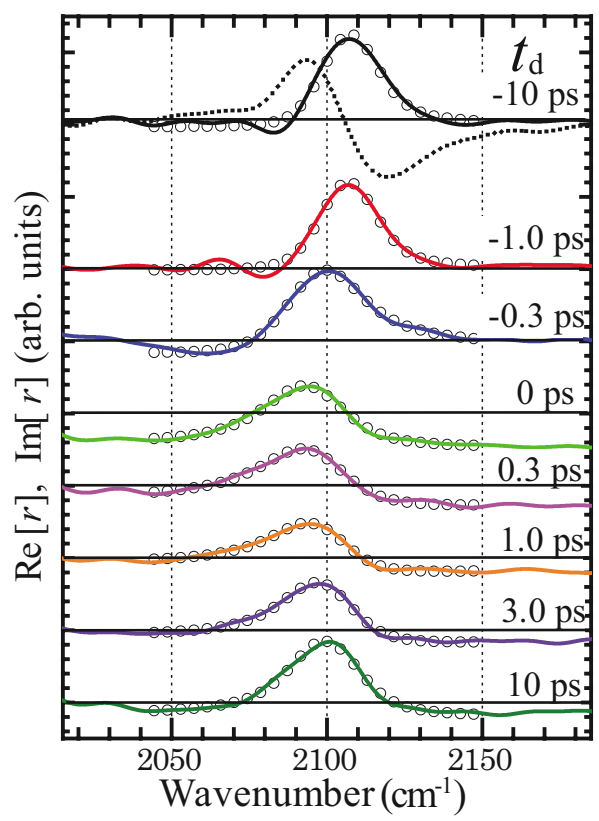

FIG. 2. (Color online) Typical $t_{\mathrm{d}}$ dependence of real (dotted line) and imaginary (solid lines) part of $r(\omega)$. The real part is shown only for $t_{\mathrm{d}}=-10 \mathrm{ps}$. The absorbed pump fluence was $13 \mathrm{~mJ} / \mathrm{cm}^{2}$. The horizontal axis is converted to $\omega_{\mathrm{IR}}$. Open circles are fitting results by using Eq. (2).

$\gamma$ are the Raman tensors, the dynamic dipole, the vibrational resonance frequency, and the dephasing rate of the $\mathrm{C}-\mathrm{O}$ mode, respectively. ${ }^{30}$ The spectrum of $\operatorname{Im}\left[\chi_{R}^{(2)}\right]$ gives a Lorentzian line shape if all the parameters are real. According to Langreth, ${ }^{24}$ however, $\mu$ is complex: $\mu=\mu_{1}+i \mu_{2}$, whenever the damping of a vibrational mode is caused by EHP excitation. This imaginary part $\mu_{2}$ introduces asymmetry in linear infrared absorption ${ }^{24}$ as well as $\operatorname{Im}\left[\chi_{\mathrm{R}}^{(2)}\right]$ spectral lines. Defining the asymmetric parameter $\tau$ as $\omega_{\mathrm{IR}} \tau=\mu_{2} / \mu_{1}$, we obtain

$$
\operatorname{Im}\left[\chi_{\mathrm{s}}^{(2)}\right]=A^{\prime} \frac{\omega_{\mathrm{IR}} \tau\left(\omega_{\mathrm{IR}}-\omega_{0}\right)-\gamma}{\left(\omega_{\mathrm{IR}}-\omega_{0}\right)^{2}+\gamma^{2}}+\delta \chi_{\mathrm{NR}}
$$

where the first term is $\operatorname{Im}\left[\chi_{\mathrm{R}}^{(2)}\right]$ with a constant factor $A^{\prime}$, and $\delta \chi_{\mathrm{NR}}$ is $\operatorname{Im}\left[\chi_{\mathrm{NR}}^{(2)}\right]$ that is assumed to be frequency independent in the IR range probed. Convolution of Eq. (2) with an instrument response function (a Gaussian with a full width of $\left.17 \mathrm{~cm}^{-1}\right)$ was used to fit $\operatorname{Im}\left[r\left(\omega_{\mathrm{IR}}\right)\right]$.

Fitting results are plotted in Fig. 2 and the optimum values of parameters: $\delta \chi_{\mathrm{NR}}, \tau, \gamma$, and $\delta \omega_{0}\left(t_{\mathrm{d}}\right)=\omega_{0}\left(t_{\mathrm{d}}\right)-\omega_{0}$ $(-10 \mathrm{ps})$, are plotted in Fig. 3 for various pump fluence conditions. Figure 3(a) shows comparison of the time evolutions of $\delta \chi_{\mathrm{NR}}$ with those of $T_{\mathrm{e}}$ calculated by using the two temperature models where coupled thermal diffusion equations for electrons and phonons are numerically integrated with parameters available in the literature. ${ }^{8,31}$ At $t_{\mathrm{d}} \sim 0 \mathrm{ps}$, the modulus of $\delta \chi_{\mathrm{NR}}$ sharply increases and decays. The transient changes in $-\delta \chi_{\mathrm{NR}}$ follow those in $T_{\mathrm{e}}$. Moreover, as in Fig. $4(\mathrm{a})$, all the data of $\delta \chi_{\mathrm{NR}}$ obtained under various pump fluence conditions show a good correlation with calculated $T_{\mathrm{e}}$. This firmly establishes the fact that the change in $\delta \chi_{\mathrm{NR}}$ is 


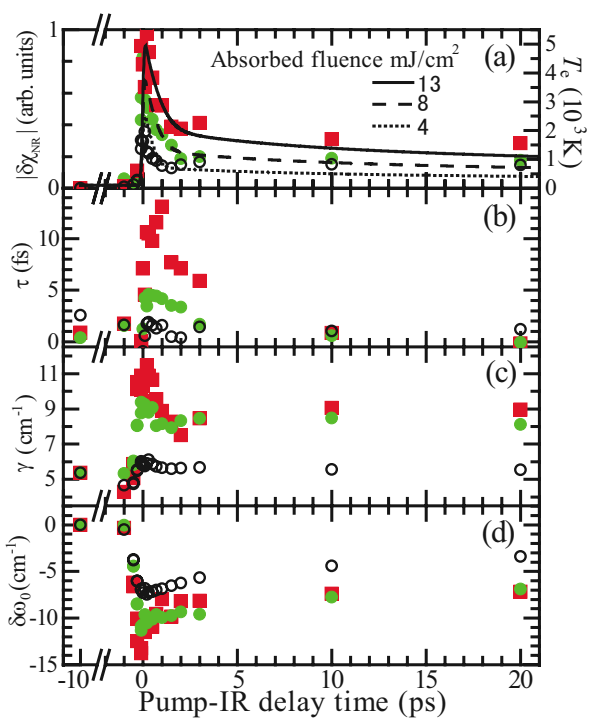

FIG. 3. (Color online) Parameters obtained by fitting $\operatorname{Im}[r(\omega)]$ with Eq. (2): (a) nonresonant background $\delta \chi_{\mathrm{NR}}$, (b) the asymmetric parameter $\tau$, (c) the dephasing rate $\gamma$, and (d) the resonance frequency shift $\delta \omega_{0}$. Absorbed fluences were 13 (red square), 8 (green filled circle), and $4 \mathrm{~mJ} / \mathrm{cm}^{2}$ (black open circle). Solid, dashed, and dotted curves in (a) are surface electronic temperature $T_{\mathrm{e}}$ calculated with the two temperature model for absorbed fluences of $13 \mathrm{~mJ} / \mathrm{cm}^{2}, 8 \mathrm{~mJ} / \mathrm{cm}^{2}$, and $4 \mathrm{~mJ} / \mathrm{cm}^{2}$, respectively. Experimental errors are estimated to be \pm 1.8 fs for $\tau, \pm 0.8 \mathrm{~cm}^{-1}$ for $\gamma$, and $\pm 0.5 \mathrm{~cm}^{-1}$ for $\omega_{0}$. Errors for $\delta \chi_{\mathrm{NR}}$ are $\pm 5 \%$ of the maximum modulation magnitude at $I=13 \mathrm{~mJ} / \mathrm{cm}^{2}$.

governed by thermally distributed hot electrons; hence, it provides a good indicator of $T_{\mathrm{e}}$.

Asymmetric parameter $\tau$ also increases sharply at $t_{\mathrm{d}}$ $\sim 0$ ps particularly at $I \geq 8 \mathrm{~mJ} / \mathrm{cm}^{2}$ [Fig. 3(b)]. Sorbello showed that $\tau$ is related to the $q$ parameter of Fano line shape: $q=-\left(\omega_{0} \tau\right)^{-1} .^{32,23}$ The $q$ parameter is determined by various factors, including the Fermi-Dirac distribution function $f_{k}$, the electron-phonon coupling constant $g_{k k^{\prime}}$, the coupling of the electronic system to an IR field $m_{k k^{\prime}}^{\prime}$, and the

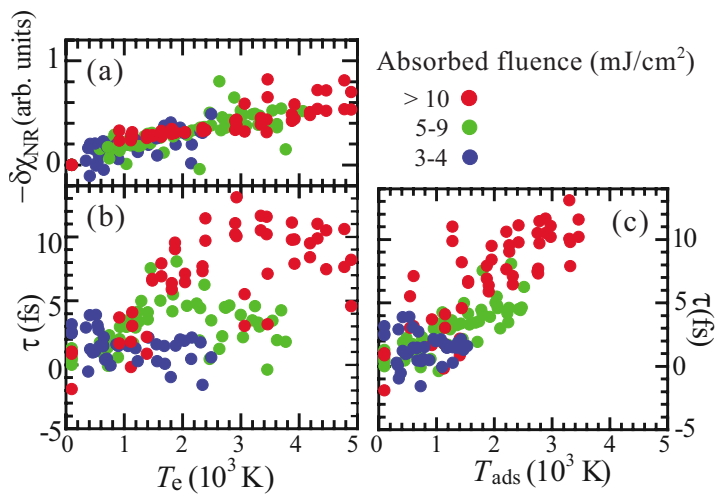

FIG. 4. (Color online) Plots of (a) vibrationally nonresonant background $\delta \chi_{\mathrm{NR}}$ vs substrate electron temperature $T_{\mathrm{e}}$, (b) $\tau$ vs $T_{\mathrm{e}}$, and (c) $\tau$ vs adsorbate temperature $T_{\mathrm{a}}$ estimated by using $\eta_{\mathrm{e}}$ $=1 / 0.4 \mathrm{ps}^{-1}$. The color code indicates different fluence ranges denoted in the figure. Data sets of $\delta \chi_{\mathrm{NR}}$ with different fluence conditions are normalized at $2000 \mathrm{~K}$. electron-state energy $\epsilon_{k}$. Here, the electron states are labeled by $k$ and $k^{\prime}$. Among these factors, $f_{k}$ and $g_{k k^{\prime}}$ can depend on temperature.

If the changes in $\tau$ are caused solely by $f_{k}, \tau$ should be a single-valued function of $T_{\mathrm{e}}$. However, a plot of $\tau$ against $T_{\mathrm{e}}$ at various pump fluences [Fig. 4(b)] shows that $\tau$ spreads widely in contrast to $\delta \chi_{\mathrm{NR}} ; \tau$ does not show a good correlation with $T_{\mathrm{e}}$. This implies that the transient changes in $\tau$ are not driven solely by substrate electron temperature.

Because the coupling of substrate electrons to an adsorbate vibrational mode depends on its normal-mode coordinate, excitation of the vibrational mode modulates $g_{k k^{\prime}}{ }^{33}$ Thus, the electron-phonon coupling will correlate with adsorbate temperature $T_{\mathrm{a}}$ rather than $T_{\mathrm{e}}$ under the assumption that the ensemble of adsorbates is thermalized. The time dependence of $T_{\mathrm{a}}$ is usually assumed to satisfy the heat-transfer equation, $d T_{\mathrm{a}} / d t=\eta_{\mathrm{e}}\left[T_{\mathrm{e}}(t)-T_{\mathrm{a}}(t)\right]$, where $\eta_{\mathrm{e}}$ is the electronfriction coefficient. Assuming that electron-phonon coupling is independent of $T_{\mathrm{e}}$ for simplicity, we replotted $\tau$ against $T_{\mathrm{a}}$ by changing $1 / \eta_{\mathrm{e}}$ in the range from 0.1 to $2 \mathrm{ps}$. This resulted in that the variance of $\tau$ vs $T_{\mathrm{a}}$ was minimized at $1 / \eta_{\mathrm{e}}$ $=0.4$ ps, as shown in Fig. 4(c); clearly, $\tau$ is better correlated with $T_{\mathrm{a}}$ than with $T_{\mathrm{e}}$.

The enhancement of nonadiabatic coupling also manifests itself in the time evolution of $\gamma$ [Fig. 3(c)]. The golden rule gives an electronic damping rate $\gamma_{\mathrm{e}}=\frac{2 \pi}{\hbar} \Sigma_{k, k^{\prime}}\left|g_{k k^{\prime}}\right|^{2} f_{k^{\prime}}(1$ $\left.-f_{k}\right) \delta\left(\hbar \omega-\epsilon_{k}+\epsilon_{k^{\prime}}\right) ;{ }^{32}$ hence, it also depends on $g_{k k^{\prime}}$. In addition to $\gamma_{\mathrm{e}}$, pure dephasing caused by anharmonic couplings to frustrated modes also contributes to the total dephasing rate. This makes it difficult to extract quantitatively how $\gamma_{\mathrm{e}}$ depends on temperature from the $t_{\mathrm{d}}$ dependence of $\gamma$. Nevertheless, the rapid rise and decay of $\gamma$ in $0<t_{\mathrm{d}}<2$ ps at $I$ $\geq 8 \mathrm{~mJ} / \mathrm{cm}^{2}$ appearing concomitantly with $\tau$ indicates that this fast component likely stems from the same origin of the $\tau$ enhancement, i.e., $g_{k k^{\prime}}$. On the basis of $t_{\mathrm{d}}$ dependence of $\tau$, the enhancement of nonadiabatic coupling almost ceases at $t_{\mathrm{d}} \sim 4$ ps. Thus, the dephasing by the anharmonic couplings largely contributes to the slowly decaying component of $\gamma$ at $t_{\mathrm{d}}>2$ ps.

Now we discuss which frustrated mode is most responsible for the enhancement of nonadiabatic coupling between the IS mode and substrate electrons. Because FR modes more effectively couple to substrate electrons than FT modes: $1 / \eta_{\mathrm{e}} \sim 0.1 \mathrm{ps}$ for FR vs $1-2$ ps for FT, ${ }^{11,12,26}$ the optimum value of $1 / \eta_{\mathrm{e}}=0.4$ ps suggests that the FR mode excitations play an important role in the enhancement of nonadiabatic coupling.

Possible origins for the enhancement associated with FR mode excitations are in the following. First, upon excitation along the coordinates of FR modes, the adsorbate probes regions away from the equilibrium position where the overlap between $2 \pi^{*}$ orbitals of $\mathrm{CO}$ and $\mathrm{Pt} d$ orbitals is larger, ${ }^{34}$ this may increase $g_{k k^{\prime}}$. Second, as a result of anharmonic coupling between the IS and FR modes, an indirect pathway for the nonadiabatic coupling of IS mode opens through excitation of FR modes that more strongly couple to substrate electrons than the IS mode. ${ }^{35}$

Finally we remark whether or not hot bands of C-O IS contribute to the observed asymmetry. Taking into account of lateral dipole-dipole coupling of $\mathrm{CO}$, the amount of redshift 
due to C-O hot bands is given by $4 C \omega_{e} x_{e}$, where $\omega_{e} x_{e}$ is the anharmonicity constant of C-O IS mode and $C$ is a fraction of $\mathrm{C}-\mathrm{O}$ phonons excited per $\mathrm{C}-\mathrm{O}$ oscillator. ${ }^{36}$ With a reported value of $\omega_{e} x_{e}=13.5 \mathrm{~cm}^{-1}, 3$ the shift is estimated to be at most $27 \mathrm{~cm}^{-1}$ with $C$ as large as 0.5 ; the shift is too small to explain the observed long tail extending lower than $2050 \mathrm{~cm}^{-1}$. In addition, the amplitude of $\tau$ decays much slower than the C-O population lifetime $(2.2 \mathrm{ps}){ }^{3}{ }^{3}$ the decay times of $\tau$ estimated by single exponential fit fall in $6.0 \pm 1.6 \mathrm{ps}$ at $I \geq 11 \mathrm{~mJ} / \mathrm{cm}^{2}$. Thus, the contribution of $\mathrm{C}-\mathrm{O}$ hot bands to the line-shape asymmetry is minor.

In summary, we have observed significant asymmetry in the line shape of $\operatorname{Im}\left[\chi_{s}^{(2)}\right]$ due to the Fano interference be- tween the C-O IS mode and a substrate EHP continuum under intense laser excitation of substrate electrons. The asymmetric parameter is well correlated with adsorbate temperature, indicating that subpicosecond heating of the frustrated modes are responsible for the enhancement of nonadiabatic coupling between $\mathrm{C}-\mathrm{O}$ stretching and substrate electrons.

This work was supported by the JST-PRESTO program and a Grant-in-Aid for Scientific Research (S) (Grant No. 17105001) from the Japanese Society for the Promotion of Sciences. The authors thank Tomokazu Yasuike for fruitful discussions. *kw@kuchem.kyoto-u.ac.jp

${ }^{1}$ J. W. Gadzuk and A. C. Luntz, Surf. Sci. 144, 429 (1984).

${ }^{2}$ J. C. Tully, Annu. Rev. Phys. Chem. 51, 153 (2000).

${ }^{3}$ J. D. Beckerle, R. R. Cavanagh, M. P. Casassa, E. J. Heilweil, and J. C. Stephenson, J. Chem. Phys. 95, 5403 (1991).

${ }^{4}$ M. Morin, N. J. Levinos, and A. L. Harris, J. Chem. Phys. 96, 3950 (1992)

${ }^{5}$ B. N. J. Persson and M. Persson, Solid State Commun. 36, 175 (1980).

${ }^{6}$ B. Hellsing and M. Persson, Phys. Scr. 29, 360 (1984).

${ }^{7}$ C. Frischkorn and M. Wolf, Chem. Rev. 106, 4207 (2006).

${ }^{8}$ T. A. Germer, J. C. Stephenson, E. J. Heilweil, and R. R. Cavanagh, J. Chem. Phys. 98, 9986 (1993).

${ }^{9}$ S. Katano, S. Dobashi, J. Kubota, K. Onda, A. Wada, S. S. Kano, and K. Domen, Chem. Phys. Lett. 377, 601 (2003).

${ }^{10}$ M. Bonn, C. Hess, S. Funk, J. H. Miners, B. N. J. Persson, M. Wolf, and G. Ertl, Phys. Rev. Lett. 84, 4653 (2000).

${ }^{11}$ F. Fournier, W. Zheng, S. Carrez, H. Dubost, and B. Bourguignon, J. Chem. Phys. 121, 4839 (2004).

${ }^{12}$ E. H. G. Backus, A. Eichler, A. W. Kleyn, and M. Bonn, Science 310, 1790 (2005).

${ }^{13}$ I. M. Lane, Z.-P. Liu, D. A. King, and H. Arnolds, J. Phys. Chem. C 111, 14198 (2007).

${ }^{14}$ B. N. J. Persson and R. Ryberg, Phys. Rev. B 32, 3586 (1985).

${ }^{15}$ J. A. Prybyla, T. F. Heinz, J. A. Misewich, M. M. T. Loy, and J. H. Glownia, Phys. Rev. Lett. 64, 1537 (1990).

${ }^{16}$ L. M. Struck, L. J. Richter, S. A. Buntin, R. R. Cavanagh, and J. C. Stephenson, Phys. Rev. Lett. 77, 4576 (1996).

${ }^{17}$ M. Bonn, S. Funk, C. Hess, D. N. Denzler, C. Stempfl, M. Scheffler, M. Wolf, and G. Ertl, Science 285, 1042 (1999).

${ }^{18}$ D. N. Denzler, C. Frischkorn, C. Hess, M. Wolf, and G. Ertl,
Phys. Rev. Lett. 91, 226102 (2003).

${ }^{19}$ L. Bartels, F. Wang, D. Möller, E. Knoesel, and T. F. Heinz, Science 305, 648 (2004).

${ }^{20}$ M. Lawrenz, K. Stépán, J. Güdde, and U. Höfer, Phys. Rev. B 80, 075429 (2009).

${ }^{21}$ M. Brandbyge, P. Hedegard, T. F. Heinz, J. A. Misewich, and D. M. Newns, Phys. Rev. B 52, 6042 (1995).

${ }^{22}$ N. Ji, V. Ostroverkhov, C. S. Tian, and Y. R. Shen, Phys. Rev. Lett. 100, 096102 (2008).

${ }^{23}$ U. Fano, Phys. Rev. 124, 1866 (1961).

${ }^{24}$ D. C. Langreth, Phys. Rev. Lett. 54, 126 (1985).

${ }^{25}$ Z. Crljen and D. C. Langreth, Phys. Rev. B 35, 4224 (1987).

${ }^{26}$ M. Nagao, K. Watanabe, and Y. Matsumoto, J. Phys. Chem. C 113, 11712 (2009).

${ }^{27}$ I. Stiopkin, H. Jayathilake, A. Bordenyuk, and A. Benderskii, J. Am. Chem. Soc. 130, 2271 (2008).

${ }^{28}$ S. Nihonyanagi, S. Yamaguchi, and T. Tahara, J. Chem. Phys. 130, 204704 (2009).

${ }^{29}$ L. Lepetit, G. Chériaux, and M. Joffre, J. Opt. Soc. Am. B 12, 2467 (1995).

${ }^{30}$ J. H. Hunt, P. Guyot-Sionnest, and Y. R. Shen, Chem. Phys. Lett. 133, 189 (1987).

${ }^{31}$ S. I. Anisimov, B. L. Kapeliovich, and T. L. Perel'man, Sov. Phys. JETP 39, 375 (1974).

${ }^{32}$ R. S. Sorbello, Phys. Rev. B 32, 6294 (1985).

${ }^{33}$ B. N. J. Persson and H. Ueba, Phys. Rev. B 76, 125401 (2007).

${ }^{34}$ J. T. Kindt, J. C. Tully, M. Head-Gordon, and M. A. Gomez, J. Chem. Phys. 109, 3629 (1998).

${ }^{35}$ H. Ueba and B. N. J. Persson, Phys. Rev. B 77, 035413 (2008).

${ }^{36}$ J. C. Kimball, C. Y. Fong, and Y. R. Shen, Phys. Rev. B 23, 4946 (1981). 